\title{
Tecnología, un buen aliado para los tiempos de pandemia
}

\section{Technology, a good ally for times of pandemic}

1 María Alejandrina Nivela Cornejo

(iD) http://orcid.org/0000-0002-0356-7243

Universidad de Guayaquil, Guayaquil, Ecuador

maria.nivelac@ug.edu.ec

2 Segundo Vicente Echeverría Desiderio

(iD) http://orcid.org/0000-0002-0235-190X

Universidad de Guayaquil, Guayaquil, Ecuador

segundo.echeverriad@ug.edu.ec

3 Carlota Alexandra Tenesaca Morales

Universidad de Guayaquil, Guayaquil, Ecuador

https://orcid.org/0000-0002-6007-0532

carlota.tenesacam@ug.edu.ec

Artículo de Investigación Científica y Tecnológica

Enviado: 08/12/2021

Revisado: $23 / 12 / 2021$

Aceptado: 28/01/2022

Publicado:05/02/2022

DOI: https://doi.org/10.33262/ap.v4i1.1.155 \section{Tecnología, un buen aliado para los tiempos de pandemia . AlfaPublicaciones, 4(1.1), 208- \\ Cítese: $\quad$ 233. https://doi.org/10.33262/ap.v4i1.1.155}

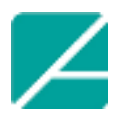

Ciencia

Digital

(i) (9)

ALFA PUBLICACIONES, es una Revista Multidisciplinar, Trimestral, que se publicará en soporte electrónico tiene como misión contribuir a la formación de profesionales competentes con visión humanística y crítica que sean capaces de exponer sus resultados investigativos y científicos en la misma medida que se promueva mediante su intervención cambios positivos en la sociedad. https://alfapublicaciones.com

La revista es editada por la Editorial Ciencia Digital (Editorial de prestigio registrada en la Cámara Ecuatoriana de Libro con No de Afiliación 663) www.celibro.org.ec

Esta revista está protegida bajo una licencia Creative Commons Attribution Non Commercial No Derivatives 4.0 International. Copia de la licencia: http://creativecommons.org/licenses/by-nc-nd/4.0/ 


\section{Palabras claves:}

tecnología, herramientas tecnológicas, superior educación, pandemia, covid-19.

\section{Resumen}

Este estudio tuvo por objetivo describir como la tecnología se ha convertido en un aliado en tiempos de pandemia para los estudiantes de la carrera de Literatura de la Facultad de Filosofía, Letras y Ciencias de la Educación. Para ello, metodológicamente se desarrolló una investigación cuantitativa, de tipo descriptiva, exploratoria, con diseño de campo, no experimental, transversal. La población fue de 120 estudiantes inscritos en la carrera de literatura de la Facultad de Filosofía, Letras y Ciencias de la Educación, Universidad de Guayaquil. Como técnica para recolección de datos se usó la encuesta, mediante la aplicación de un cuestionario, aplicado a través de Google Forms, con 17 proposiciones de respuestas cerradas, distribuidas en dos dimensiones: tipos de herramientas tecnológicas y uso de la tecnología, estas constituidas por cinco subdimensiones. Como resultados se tienen para el acceso a la información, una tendencia hacia el mucho uso de Laptot o PC, Tablets y teléfonos inteligentes; para la conectividad y coordinación de tareas, se evidencia una tendencia hacia el mucho uso de internet y wi-fi; sin embargo, hacen uso medio de Bluetooth; para tecnologías expositivas, se evidencia que la mayoría de los estudiantes prefiere usar Power Point a Prezi; para las Plataformas Tecnológicas, se evidencia que la mayoría de los estudiantes prefieren usar mucho Moodle, Zoom y Google Meet; entre las aplicaciones, se evidencia que la mayoría hacen mucho uso de: YouTube, WhatsApp y Telegram; y finalmente la mayoría de los aprendices, un $85.8 \%$ opinó que los beneficios del uso de la tecnología son muchos. En conclusión, la tecnología ha sido un buen aliado para los tiempos de pandemia.

\section{Keywords: \\ technology, technological tools, higher education, pandemic, covid-19.}

\begin{abstract}
This study aimed to describe how technology has become an ally in times of pandemic for students of the Literature career of the Faculty of Philosophy, Letters and Education Sciences. For this, a quantitative, descriptive, exploratory, field design, nonexperimental, cross-sectional study was methodologically developed. The population was 120 students enrolled in the literature career of the Faculty of Philosophy, Letters and Educational Sciences, University of Guayaquil. As a data collection technique, the survey was used, through the application of a
\end{abstract}


questionnaire, applied through Google Forms, with 17 propositions of closed answers, distributed in two dimensions: types of technological tools and use of technology, these constituted by five subdimensions. As results, for access to information, there is a tendency towards the extensive use of Laptops or PCs, Tablets and smartphones; for connectivity and coordination of tasks, there is evidence of a trend towards extensive use of the internet and wi-fi; however, they make medium use of Bluetooth; For expository technologies, it is evident that most of the ex-students prefer to use Power Point to Prezi; For Technological Platforms, it is evident that most of the students prefer to use Moodle, Zoom and Google Meet a lot; Among the applications, it is evident that the majority make a lot of use of: YouTube, WhatsApp and Telegram; and finally the majority of the apprentices, $85.8 \%$ thought that the benefits of using technology are many. In conclusion, technology has been a good ally in times of pandemic

\section{Introducción}

Debido a la pandemia declarada por la organización mundial de la salud en el 2020, los gobiernos del mundo tomaron medidas sanitarias entre las que se incluyó la suspensión de las clases presenciales y la utilización de tecnologías para el desarrollo virtual de las actividades educativas; esta acción para la cual ninguna institución educativa estaba preparada, ha develado fortalezas y dificultades de los sistemas educativos con respecto al uso de las bondades y beneficios que ofrecen las tecnologías en general al proceso de enseñanza y aprendizaje.

La tecnología ha permeado la vida del ser humano al hacerse indispensable para su desarrollo individual. En tiempos de pandemia ellas han ofrecido un conjunto de soluciones que han permitido a las personas permanecer en sus hogares, disminuyendo así el riesgo de contagio, entre estas se encuentran las aplicaciones o apps, webs y chatbots que implementan test de preguntas y respuestas, consultas de información, o incluso registro de citas previas en distintos servicios.

Durante el confinamiento declarado por causa de la pandemia del Covid-19, las herramientas tecnológicas que más han sido usadas para la comunicación y las actividades educativas han sido las videoconferencias, a través de Zoom, Google Meet, Teams, Hang Outs y otras, las cuales, debido al incremento en su uso, las empresas se vieron obligadas a mejorar su tecnología para hacerlas más accesibles y amigables. Algunos docentes, sin embargo, optaron por organizar sus actividades mediante tareas por grupos de WhatsApp 
o Telegram, el envío de documentos en PDF, Word e imágenes con ejercicios descartando la posibilidad de clases por videollamada y actividades sincrónicas (Linne, 2020).

En la educación superior las tecnologías de información y comunicación (TIC) han sido herramientas indispensables, debido a su contribución para la interrelación entre los actores principales del acto educativo; es decir, docentes y aprendices, han facilitado a los primeros desde la acción de planificar las estrategias hasta la puesta en práctica de estas, también han ayudado a facilitar el aprendizaje de los estudiantes (Nivela et., 2021).

Son muchos los procesos educativos que se han logrado optimizar con la intervención de la tecnología, tal es el caso por ejemplo del diseño de recursos instruccionales y de enseñanza personalizados, evaluación y seguimiento de estudiantes, comunicación interactiva entre aprendices y entre estos y sus docentes, entre otros (Gil, 2019). La implementación de tecnologías en educación como herramientas para lograr el aprendizaje han sido nombradas TAC (Tecnologías para el Aprendizaje y el Conocimiento), dentro de ellas se incluye el uso de la computadora, la tableta, el celular e incluso los robots (Ghitis \& Alba, 2019).

Existen variadas estrategias para trabajar en ambientes educativos mediados por tecnologías; a continuación se detallan cuatro de ellas, la primera está vinculada a un entorno virtual de aprendizaje, se apoya el proceso de formación mediante del uso de páginas web y tutoriales; la segunda incluye la tecnología dentro del aula a través de los aparatos electrónicos, como calculadoras, computadoras, tabletas, celulares, simuladores, entre otros; la tercera se refiere al aprendizaje colaborativo mediado por plataformas virtuales que incluyen chat, foros, juegos y videoconferencias; y por último, la simulación para el aprendizaje de habilidades prácticas (Galvis, 2004).

En las instituciones de educación superior del Ecuador tanto docentes como estudiantes cuentan, sobre todo a partir del año 2020, con variadas herramientas tecnológicas, entre ellas se tienen cuatro tipos: a) las de acceso a la información, tales como: laptop, tablets, Smartphone; b) las de conectividad y coordinación de tareas: bluetooth, internet, intranet, Wi-Fi; c) las expositivas: Prezi, Power Point, Youtube y d) las plataformas tecnológicas: Moodle, buscadores como Google académico, google libros, entre otros. Además, se cuenta con entornos virtuales de formación tales como: WebCT, Learning Space, Edustance; herramientas de autor, como: Hot Potatoes, y Clic; y software tales como: Perception y Quiz Factory (sic) (Espinel, 2020).

En la Universidad de Guayaquil se han utilizado diversas tecnologías durante el tiempo de pandemia, sin embargo, es oportuno preguntarse: ¿qué tan beneficioso ha sido su uso? Ante tal interrogante surge la presente investigación, cuyo objetivo consistió en describir como la tecnología se ha convertido en un aliado en tiempos de pandemia para los 
estudiantes de la carrera de Literatura de la Facultad de Filosofía, Letras y Ciencias de la Educación.

\section{Metodología}

Esta investigación se realizó bajo el enfoque cuantitativo, fue de tipo descriptiva, según su alcance fue exploratoria, tal como lo plantean Hernández et al. (2014). El diseño asumido fue de campo, no experimental, transversal (Hurtado, 2012).

La población fue de 120 estudiantes inscritos en la carrera de literatura de la Facultad de Filosofía, Letras y Ciencias de la Educación, Universidad de Guayaquil, seleccionando la totalidad de ellos por ser una población finita y de pocos individuos. Como técnica para recolección de datos se usó la encuesta, mediante la aplicación de un cuestionario, aplicado a través de Google Forms, el mismo quedó constituido por 17 proposiciones de respuestas cerradas, con tres alternativas de selección: Mucho (3), Medio (2) y Poco (1); distribuidas en dos dimensiones: la primera, llamada: tipos de herramientas tecnológicas; la segunda: Uso de la tecnología. Las dos dimensiones constituidas por cinco subdimensiones. Este cuestionario fue validado por cinco expertos en educación cuya trayectoria es demostrada en el área del uso de la tecnología, también fue determinada su confiabilidad, obteniendo un valor de 0.81 para el Coeficiente Alfa de Cronbach, el cual es considerado muy alto según Hernández et al. (2014).

La presentación de resultados y el análisis de los datos se realizó mediante la utilización de estadística descriptiva, usando la tabla de distribución de frecuencias, con sus respectivos porcentajes. Para el procesamiento de los datos se aplicó el programa estadístico SPSS 25.

\section{Resultados y Discusión}

A continuación, se presentan los resultados estructurados por dimensiones y subdimensiones. Luego se realiza la discusión de los mismos. Para la dimensión tipos de herramientas tecnológicas, subdimensiones 1: acceso a la información, se presentan las tablas 1 a la 3 con las frecuencias y porcentajes correspondientes a las respuestas de cada ítem.

Tabla 1

Frecuencias y porcentajes de las respuestas del ítem 1: uso de Laptop o PC

\begin{tabular}{|c|c|c|c|c|c|}
\hline & & Frecuencia & Porcentaje & $\begin{array}{c}\text { Porcentaje } \\
\text { válido }\end{array}$ & $\begin{array}{l}\text { Porcentaje } \\
\text { acumulado }\end{array}$ \\
\hline \multirow[t]{4}{*}{ Válidos } & Mucho (3) & 117 & 97.5 & 97.5 & 97.5 \\
\hline & Medio (2) & 2 & 1.7 & 1.7 & 99.2 \\
\hline & Poco (1) & 1 & 0.8 & 0.8 & 100.0 \\
\hline & Total & 120 & 100.0 & 100.0 & \\
\hline
\end{tabular}


Como puede observarse en la tabla 1, para el ítem 1, la mayoría de los estudiantes, un 97.5\% opinó que usa mucho la Laptop o PC. En esta subdimensiones se presenta también la tabla 2, con las respuestas dadas al ítem 2.

Tabla 2

Frecuencias y porcentajes de las respuestas del ítem 2: uso de Tablet

\begin{tabular}{llrrrr}
\hline & & & Porcentaje & Porcentaje \\
válido & acumulado \\
\hline Válidos & Mucho (3) & 112 & 93.3 & 93.3 & 93.3 \\
& Medio (2) & 7 & 5.8 & 5.8 & 99.2 \\
& Poco (1) & 1 & 0.8 & 0.8 & 100.0 \\
\hline & Total & 120 & 100.0 & 100.0 & \\
\hline
\end{tabular}

Tal como se evidencia en la tabla 2, para el ítem 2 la mayoría de los estudiantes, un 93.3\% de ellos manifestó que usa mucho su tablet. A continuación, se presenta la tabla 3 con las respuestas de los participantes para el ítem 3 .

\section{Tabla 3}

Frecuencias y porcentajes de las respuestas del ítem 3: uso de teléfono inteligente

\begin{tabular}{|c|c|c|c|c|c|}
\hline & & Frecuencia & Porcentaje & $\begin{array}{l}\text { Porcentaje } \\
\text { válido }\end{array}$ & $\begin{array}{l}\text { Porcentaje } \\
\text { acumulado }\end{array}$ \\
\hline \multirow[t]{4}{*}{ Válidos } & Mucho (3) & 115 & 95.8 & 95.8 & 95.8 \\
\hline & Medio (2) & 3 & 2.5 & 2.5 & 98.3 \\
\hline & Poco (1) & 2 & 1.7 & 1.7 & 100.0 \\
\hline & Total & 120 & 100.0 & 100.0 & \\
\hline
\end{tabular}

Como puede observarse en la tabla 3, en el ítem 3 un 95.8\% de los estudiantes expresó que usa mucho su teléfono inteligente. De esta forma, para el subdimensión: acceso a la información, se evidencia una tendencia hacia el mucho uso de estos tres dispositivos; es decir, Laptop o PC, Tablets y teléfonos inteligentes.

Para el subdimensión 2: conectividad y coordinación de tareas, se presentan las tablas 4 a la 6 con las frecuencias y porcentajes correspondientes a las respuestas de cada ítem.

\section{Tabla 4}

Frecuencias y porcentajes de las respuestas del item 4: uso de Bluetooth

\begin{tabular}{llrrrr}
\hline & & & \multicolumn{2}{c}{$\begin{array}{c}\text { Porcentaje } \\
\text { válido }\end{array}$} & $\begin{array}{r}\text { Porcentaje } \\
\text { acumulado }\end{array}$ \\
\hline Válidos & Mucho (3) & 15 & 12.5 & 12.5 & 12.5 \\
& Medio (2) & 72 & 60.0 & 60.0 & 72.5 \\
& Poco (1) & 33 & 27.5 & 27.5 & 100.0 \\
\cline { 2 - 6 } & Total & 120 & 100.0 & 100.0 &
\end{tabular}


En la tabla 4 se muestra para el ítem 4 que un $60 \%$ de los estudiantes expresó que hacen uso medio de Bluetooth, observando además una tendencia del $33 \%$ hacia el poco uso del mismo. Seguidamente se presenta la tabla 5, con las frecuencias y porcentajes de las respuestas dadas al ítem 5.

\section{Tabla 5}

Frecuencias y porcentajes de las respuestas del ítem 5: uso de Wi-Fi

\begin{tabular}{llrrrr}
\hline & & & \multicolumn{2}{c}{$\begin{array}{c}\text { Porcentaje } \\
\text { válido }\end{array}$} & $\begin{array}{c}\text { Porcentaje } \\
\text { acumulado }\end{array}$ \\
\hline Válidos & Mucho (3) & 113 & 94.2 & 94.2 & 94.2 \\
& Medio (2) & 5 & 4.2 & 4.2 & 98.3 \\
& Poco (1) & 2 & 1.7 & 1.7 & 100.0 \\
\cline { 2 - 6 } & Total & 120 & 100.0 & 100.0 & \\
\hline
\end{tabular}

Tal como se muestra en la tabla 5, un $94.2 \%$ de los estudiantes manifestó que hace mucho uso del Wi-Fi. A continuación, se presenta la tabla 6 con las frecuencias y porcentajes de respuestas de los estudiantes para el ítem 6.

\section{Tabla 6}

Frecuencias y porcentajes de las respuestas del ítem 6: uso de Internet

\begin{tabular}{|c|c|c|c|c|c|}
\hline & & Frecuencia & Porcentaje & $\begin{array}{l}\text { Porcentaje } \\
\text { válido }\end{array}$ & $\begin{array}{l}\text { Porcentaje } \\
\text { acumulado }\end{array}$ \\
\hline \multirow[t]{4}{*}{ Válidos } & Mucho (3) & 120 & 100.0 & 100.0 & 100.0 \\
\hline & Medio (2) & 0 & 0.0 & 0.0 & 100.0 \\
\hline & Poco (1) & 0 & 0.0 & 0.0 & 100.0 \\
\hline & Total & 120 & 100.0 & 100.0 & \\
\hline
\end{tabular}

Como puede observarse en la tabla 6, en el ítem 6 un 100\% de los estudiantes expresó que usa mucho el internet. De esta forma, para los subdimensiones: conectividad y coordinación de tareas, se evidencia una tendencia hacia el mucho uso de internet y Wifi; sin embargo, hacen uso medio de Bluetooth.

Para los subdimensiones 3: tecnologías expositivas, se presentan las tablas 7 y 8 con las frecuencias y porcentajes correspondientes a las respuestas de cada ítem.

\section{Tabla 7}

Frecuencias y porcentajes de las respuestas del ítem 7: uso de Power Point

\begin{tabular}{llrrrr}
\hline & & & Porcentaje & Porcentaje \\
válido & Fcumulado \\
\hline Válidos & Mucho (3) & 113 & 94.2 & 94.2 & 94.2 \\
& Medio (2) & 5 & 4.2 & 4.2 & 98.3 \\
& Poco (1) & 2 & 1.7 & 1.7 & 100.0 \\
\cline { 2 - 6 } & Total & 120 & 100.0 & 100.0 & \\
\hline
\end{tabular}


En la tabla 7 se puede evidenciar que la mayoría de los estudiantes, un 94.2\%, expresó que usan mucho el Power Point como herramienta de presentación para sus exposiciones. Seguidamente se presenta la tabla 8 con los porcentajes y frecuencias de las respuestas dadas al ítem 8 por parte de los encuestados.

\section{Tabla 8}

Frecuencias y porcentajes de las respuestas del ítem 8: uso de Prezi

\begin{tabular}{llrrrr}
\hline & & & \multicolumn{2}{c}{$\begin{array}{c}\text { Porcentaje } \\
\text { válido }\end{array}$} & $\begin{array}{l}\text { Porcentaje } \\
\text { acumulado }\end{array}$ \\
\hline Válidos & Mucho (3) & 57 & 47.5 & 47.5 & 47.5 \\
& Medio (2) & 46 & 38.3 & 38.3 & 85.8 \\
& Poco (1) & 15 & 12.5 & 12.5 & 98.3 \\
\cline { 2 - 6 } & Total & 118 & 98.3 & 98.3 & \\
\hline
\end{tabular}

Como se puede observar en la tabla 8 , la mayoría de los participantes de la investigación, un $47.5 \%$ hacen mucho uso de Prezi, seguidos de un $38.3 \%$ que hacen uso medio y un $12.5 \%$ que hacen poco uso de esta herramienta expositiva. De esta forma, para la subdimensión: tecnologías expositivas, se evidencia que la mayoría de los estudiantes prefiere usar Power Point a Prezi.

Para la subdimensión 4: Plataformas tecnológicas, se presentan las tablas 9 a la 13 con las frecuencias y porcentajes correspondientes a las respuestas de cada ítem.

\section{Tabla 9}

Frecuencias y porcentajes de las respuestas del ítem 9: uso de Moodle

\begin{tabular}{llrrrr}
\hline & & & & Porcentaje & \multicolumn{2}{c}{$\begin{array}{c}\text { Porcentaje } \\
\text { acumulado }\end{array}$} \\
\hline Válidos & Frecuencia & Porcentaje & válido & acula \\
& Mucho (3) & 112 & 93.3 & 93.3 & 93.3 \\
& Medio (2) & 6 & 5.0 & 5.0 & 98.3 \\
& Poco (1) & 2 & 1.7 & 1.7 & 100.0 \\
\cline { 2 - 7 } & Total & 120 & 100.0 & 100.0 & \\
\hline
\end{tabular}

Tal como se videncia en la tabla 9, la mayoría de los aprendices, un 93.3\% opinaron que hacen mucho uso de Moodle como plataforma tecnológica. Seguidamente se presenta la tabla 10 con las frecuencias y porcentajes de respuestas dadas al ítem 10.

\section{Tabla 10}

Frecuencias y porcentajes de las respuestas del ítem 10: uso de Schoology

\begin{tabular}{llllll}
\hline & & Frecuencia & Porcentaje & $\begin{array}{l}\text { Porcentaje } \\
\text { válido }\end{array}$ & $\begin{array}{l}\text { Porcentaje } \\
\text { acumulado }\end{array}$ \\
\hline Válidos & Mucho (3) & 45 & 37.5 & 37.5 & 37.5 \\
& Medio (2) & 8 & 6.7 & 6.7 & 44.2 \\
& Poco (1) & 67 & 55.8 & 55.8 & 100.0 \\
\cline { 2 - 6 } & Total & 120 & 100.0 & 100.0 & \\
\hline
\end{tabular}


En la tabla 10 se muestra que la mayoría de los estudiantes, un $55.8 \%$, hacen poco uso de la plataforma Schoology. A continuación, se presenta la tabla 11, con las frecuencias y porcentajes de respuestas dadas por los participantes al ítem 11.

\section{Tabla 11}

Frecuencias y porcentajes de las respuestas del ítem 11: uso de Edmodo

\begin{tabular}{|c|c|c|c|c|c|}
\hline & & Frecuencia & Porcentaje & $\begin{array}{l}\text { Porcentaje } \\
\text { válido }\end{array}$ & $\begin{array}{l}\text { Porcentaje } \\
\text { acumulado }\end{array}$ \\
\hline \multirow[t]{4}{*}{ Válidos } & Mucho (3) & 27 & 22.5 & 22.5 & 22.5 \\
\hline & Medio (2) & 21 & 17.5 & 17.5 & 40.0 \\
\hline & Poco (1) & 71 & 59.2 & 59.2 & 99.2 \\
\hline & Total & 119 & 99.2 & 99.2 & \\
\hline
\end{tabular}

Como se muestra en la tabla 11, la mayoría de los estudiantes, un $59.2 \%$, hacen poco uso de la plataforma Edmodo. A continuación, se presenta la tabla 12, con las frecuencias y porcentajes de respuestas dadas por los participantes al ítem 12.

\section{Tabla 12}

Frecuencias y porcentajes de las respuestas del ítem 12: uso de Zoom

\begin{tabular}{llrrrr}
\hline & & & \multicolumn{2}{c}{$\begin{array}{c}\text { Porcentaje } \\
\text { válido }\end{array}$} & $\begin{array}{c}\text { Porcentaje } \\
\text { acumulado }\end{array}$ \\
\hline Válidos & Mucho (3) & 112 & 93.3 & 93.3 & 93.3 \\
& Medio (2) & 6 & 5.0 & 5.0 & 98.3 \\
& Poco (1) & 2 & 1.7 & 1.7 & 100.0 \\
\cline { 2 - 6 } & Total & 120 & 100.0 & 100.0 & \\
\hline
\end{tabular}

En la tabla 12 se muestra que la mayoría de los estudiantes, un $93.3 \%$, hacen poco uso de la plataforma Zoom. A continuación, se presenta la tabla 13, con las frecuencias y porcentajes de respuestas dadas por los participantes al ítem 13.

\section{Tabla 13}

Frecuencias y porcentajes de las respuestas del ítem 13: uso de Google Meet

\begin{tabular}{|c|c|c|c|c|c|}
\hline & & Frecuencia & Porcentaje & $\begin{array}{l}\text { Porcentaje } \\
\text { válido }\end{array}$ & $\begin{array}{l}\text { Porcentaje } \\
\text { acumulado }\end{array}$ \\
\hline \multirow[t]{4}{*}{ Válidos } & Mucho (3) & 107 & 89.2 & 89.2 & 89.2 \\
\hline & Medio (2) & 10 & 8.3 & 8.3 & 97.5 \\
\hline & Poco (1) & 3 & 2.5 & 2.5 & 100.0 \\
\hline & Total & 120 & 100.0 & 100.0 & \\
\hline
\end{tabular}

Como se evidencia en la tabla 13, la mayoría de los estudiantes, un $89.2 \%$, hacen mucho uso de la plataforma Google Meet. De esta forma, para la subdimensiones: Plataformas 
Tecnológicas, se evidencia que la mayoría de los estudiantes prefieren usar Moodle, Zoom y Google Meet en lugar de plataformas como Schoology y Edmodo.

Para la subdimensión 5: Aplicaciones, se presentan las tablas 14 a la 16 con las frecuencias y porcentajes correspondientes a las respuestas de cada ítem.

\section{Tabla 14}

Frecuencias y porcentajes de las respuestas del ítem 14: uso de WhatsApp

\begin{tabular}{llrrrr}
\hline & & & \multicolumn{2}{c}{ Porcentaje } & \multicolumn{2}{c}{$\begin{array}{c}\text { Porcentaje } \\
\text { acumulado }\end{array}$} \\
\hline Válidos & Frecuencia & Porcentaje & válido & acum \\
& Mucho (3) & 109 & 90.8 & 90.8 & 90.8 \\
& Medio (2) & 9 & 7.5 & 7.5 & 98.3 \\
& Poco (1) & 2 & 1.7 & 1.7 & 100.0 \\
\cline { 2 - 6 } & Total & 120 & 100.0 & 100.0 & \\
\hline
\end{tabular}

En la tabla 14 se evidencia que la mayoría de los estudiantes, un $90.8 \%$, hacen mucho uso de la aplicación WhatsApp. A continuación, se presenta la tabla 15, con las frecuencias y porcentajes de respuestas dadas por los participantes al ítem 15.

\section{Tabla 15}

Frecuencias y porcentajes de las respuestas del ítem 15: uso de Telegram

\begin{tabular}{|c|c|c|c|c|c|}
\hline & & Frecuencia & Porcentaje & $\begin{array}{l}\text { Porcentaje } \\
\text { válido }\end{array}$ & $\begin{array}{l}\text { Porcentaje } \\
\text { acumulado }\end{array}$ \\
\hline \multirow[t]{4}{*}{ Válidos } & Mucho (3) & 106 & 88.3 & 88.3 & 88.3 \\
\hline & Medio (2) & 11 & 9.2 & 9.2 & 97.5 \\
\hline & Poco (1) & 3 & 2.5 & 2.5 & 100.0 \\
\hline & Total & 120 & 100.0 & 100.0 & \\
\hline
\end{tabular}

Tal como se evidencia en la tabla 15 , la mayoría de los estudiantes, un $88.3 \%$, hacen mucho uso de Telegram. A continuación, se presenta la tabla 16, con las frecuencias y porcentajes de respuestas dadas por los participantes al ítem 16.

\section{Tabla 16}

Frecuencias y porcentajes de las respuestas del ítem 16: uso de YouTube

\begin{tabular}{|c|c|c|c|c|c|}
\hline & & Frecuencia & Porcentaje & $\begin{array}{l}\text { Porcentaje } \\
\text { válido }\end{array}$ & $\begin{array}{l}\text { Porcentaje } \\
\text { acumulado }\end{array}$ \\
\hline \multirow[t]{4}{*}{ Válidos } & Mucho (3) & 118 & 98.3 & 98.3 & 98.3 \\
\hline & Medio (2) & 2 & 1.7 & 1.7 & 100.0 \\
\hline & Poco (1) & 0 & 0.0 & 0.0 & 100.0 \\
\hline & Total & 120 & 100.0 & 100.0 & \\
\hline
\end{tabular}

En la tabla 16 se muestra que la mayoría de los estudiantes, un $98.3 \%$, hacen mucho uso de YouTube. De esta forma, para la subdimensiones: Aplicaciones, se evidencia que la 
mayoría hacen mucho uso de estas tres aplicaciones: WhatsApp, Telegram y YouTube.

Para la segunda dimensión: Uso de la tecnología, se presenta la tabla 17 con las frecuencias y porcentajes correspondientes a las respuestas de este ítem.

Tabla 17

Frecuencias y porcentajes de las respuestas del ítem 17: Beneficios de uso de la tecnología

\begin{tabular}{|c|c|c|c|c|c|}
\hline & & Frecuencia & Porcentaje & $\begin{array}{l}\text { Porcentaje } \\
\text { válido }\end{array}$ & $\begin{array}{l}\text { Porcentaje } \\
\text { acumulado }\end{array}$ \\
\hline \multirow[t]{4}{*}{ Válidos } & Mucho (3) & 103 & 85.8 & 85.8 & 85.8 \\
\hline & Medio (2) & 12 & 10.0 & 10.0 & 95.8 \\
\hline & Poco (1) & 5 & 4.2 & 4.2 & 100.0 \\
\hline & Total & 120 & 100.0 & 100.0 & \\
\hline
\end{tabular}

Como se evidencia en la tabla 17, la mayoría de los aprendices, un 85:8\% opinó que los beneficios del uso de la tecnología son muchos, seguido de un 10\% que expresó que son medios y un $4.2 \%$ que expresaron que son pocos.

\section{Discusión}

Con relación a los resultados obtenidos en la subdimensión 1: acceso a la información, se tiene que los mismos concuerdan con los obtenidos por Mangisch \& Mangisch (2020), quienes encontraron que el 96,9\% de los alumnos y el 97,2 \% de los profesores encuestados declararon tener y usar teléfonos inteligentes; los alumnos con un $93 \%$ consideraron el uso de sus teléfonos para las actividades universitarias entre bueno y excelente. También apoya los resultados de Parte (2021) quién encontró que el dispositivo móvil más utilizado hoy en día es el teléfono inteligente. Además, concuerda con Dafonte-Gómez et al. (2021) quienes encontraron que el $100 \%$ de la muestra usa ordenador portátil, el 98,8\% usa smartphone; el uso de los móviles es aprobado por el $89.77 \%$ como bastante útil; sin embargo, con relación al uso de tablets, estos investigadores encontraron unos resultados contrarios, según ellos el 61,4\% del alumnado no dispone y no usa tablet.

Para la subdimensión 2: conectividad y coordinación de tareas, los resultados concuerdan con los de Mangisch \& Mangisch (2020), quienes encontraron que, para la conectividad, la navegación en Internet fue la preferida por los estudiantes con el $75 \%$, al igual que Dafonte-Gómez et al. (2021) quienes encontraron que más del 80\% de los participantes de la investigación realiza búsqueda de información en Internet.

Con respecto a la subdimensión: tecnologías expositivas, los resultados concuerdan con los de Sánchez et al. (2020), quienes encontraron que los estudiantes y los docentes 
participantes prefirieron usar Power Point seguido de Prezi para sus presentaciones y realización de actividades expositivas.

Para la subdimensión: Plataformas tecnológicas se tiene que los resultados concuerdan con los obtenidos por Rojas-Londoño \& Diaz-Mora (2020) quienes encontraron que sobre las plataformas más utilizadas un 66,4\% emplean el uso de Moodle y Edmodo, y un 61,3\% usa la plataforma de Video conferencias Zoom y Google Meet. Con relación a la subdimensión: Aplicaciones, se obtuvo que los resultados concuerdan con los de Padilla et al. (2020), quienes encontraron que entre las aplicaciones que más se mencionan entre sus estudiantes están YouTube, Facebook, Messenger y WhatsApp.

Con respecto a la dimensión: Uso de la tecnología, se tiene que la mayoría de los estudiantes opinó que los beneficios del uso de esta son muchos, este resultado concuerda con los de Lagos \& Garcés (2020), quienes encontraron entre los beneficios, que un $89 \%$ de sus participantes opinó que las tecnologías fomentan el trabajo colaborativo, el 94\% consideró que flexibiliza la educación, y un 95\% expresó que permite un aprendizaje personalizado.

\section{Conclusiones}

En esta investigación se describió como la tecnología se ha convertido en un aliado en tiempos de pandemia para los estudiantes de la carrera de Literatura de la Facultad de Filosofía, Letras y Ciencias de la Educación. Esta descripción conllevó a responder la interrogante de investigación: ¿qué tan beneficioso ha sido su uso? En este sentido se tiene:

- El acceso a la información se realiza con mucha frecuencia mediante el uso de Laptop o PC, Tablets y teléfonos inteligentes, de igual forma en cuanto a tecnologías para la conectividad los estudiantes usan mayormente el internet y el wi-f.

- Para las tecnologías expositivas o de presentaciones se prefiere Power Point como herramienta, aunque también se usa el Prezi.

- Las Plataformas tecnológicas educativas preferidas están el Moodle, seguida por Zoom y Google Meet. De forma semejante, en cuanto a aplicaciones se usan en mayor medida en YouTube, WhatsApp y Telegram.

- Se destaca que los beneficios del uso de la tecnología en el proceso educativo son muchos, sobre todo a partir del confinamiento causado por la pandemia del Covid19.

- En conclusión, según estos resultados, la tecnología ha sido un buen aliado para los tiempos de pandemia. 


\section{Referencias Bibliográficas}

Dafonte-Gómez, A., Maina, M., y García-Crespo, O. (2021). Uso del smartphone en jóvenes universitarios: una oportunidad para el aprendizaje. Pixel-Bit. Revista De Medios Y Educación, 60, 211-227. https://doi.org/10.12795/pixelbit.76861

Espinel, E. (2020). La tecnología en el aprendizaje del estudiantado de la Facultad de Ciencias Químicas, Universidad Central del Ecuador. Revista Actualidades Investigativas en Educación, 20(2), 1-37. http://dx.doi.org/10.33210/ca.v8i1.209

Galvis, Á. (2004). Oportunidades educativas de las TIC. Metacursos Soluciones Elearning Innovadoras, 1-6. https://docplayer.es/4314141-Oportunidadeseducativas-de-las-tic.html

Ghitis, T. y Alba, A. (2019). Percepciones de futuros docentes sobre el uso de tecnología en educación inicial. Revista Electrónica de Investigación Educativa, 21, e23, 112. https://doi:10.24320/redie.2019.21.e23.2034

Gil, N. (2019). Ambiente virtual de aprendizaje: beneficios y ventajas para enseñanza del francés como L2. Revista Boletín REDIPE, 8(11), 91-99. https://doi.org/10.36260/rbr.v8i11.852

Lagos, G., y Garcés, E. (2020). Aplicación del U-Learning en la educación superior del Ecuador frente al COVID-19. INNOVA Research Journal, 5(3.2), 19-32. https://doi.org/10.33890/innova.v5.n3.2.2020.1600

Linne, J. (2020). Las TIC en la intersección áulica: desafíos y tensiones de la alfabetización digital en la escuela media. Revista electrónica de investigación educativa, 22, e24. Epub 28 de diciembre de 2020. https://doi.org/10.24320/redie.2020.22.e24.3072

Mangisch, G. y Mangisch, M. (2020). El uso de dispositivos móviles como estrategia educativa en la universidad. RIED. Revista Iberoamericana de Educación a Distancia, 23(1), 201-216. https://doi.org/10.5944/ried.23.1.25065

Nivela et al. (2021). Educación superior con nuevas tecnologías de información y comunicación en tiempo de pandemia. Horizontes. Revista de Investigación en Ciencias de la Educación, 519), 813-825. https://doi.org/10.33996/revistahorizontes.v5i19.239

Padilla, E., Portilla, G. y Torres, M. (2020). Aprendizaje autónomo y plataformas digitales: el uso de tutoriales de YouTube de jóvenes en Ecuador. Estudios Pedagógicos XLVI (2), 285-297. 
Parte, J. (2021). Estudio sobre aplicaciones en teléfonos inteligentes para su uso en el aula de tecnología en la Educación Secundaria Obligatoria (Trabajo fin de Máster). Universidad de Valladolid. https://uvadoc.uva.es/handle/10324/50983

Rojas-Londoño, O. y Diaz-Mora, J. (2020). COVID-19 La obligación al cambio; Transformación de la educación mediante la inclusión de herramientas tecnológicas para un aprendizaje significativo. Hamut’ay, 7 (2), 64-74. http://dx.doi.org/10.21503/hamu.v7i2.2134

Sánchez, P., Pazmiño, M. y Gámez, M. (2020). Prezi as an innovative teaching tool for the strengthening of significant learning. International Research Journal of Management, IT and Social Sciences, 7(1), 72-83. https://doi.org/10.21744/irjmis.v7n1.825

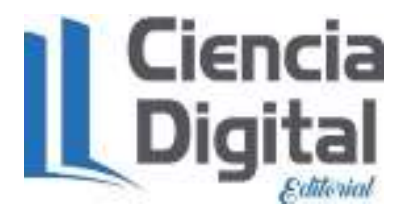


El artículo que se publica es de exclusiva responsabilidad de los autores y no necesariamente reflejan el pensamiento de la Revista Alfa Publicaciones.

\section{Ciencia}

El artículo queda en propiedad de la revista y, por tanto, su publicación parcial y/o total en otro medio tiene que ser autorizado por el director de la Revista Alfa Publicaciones.
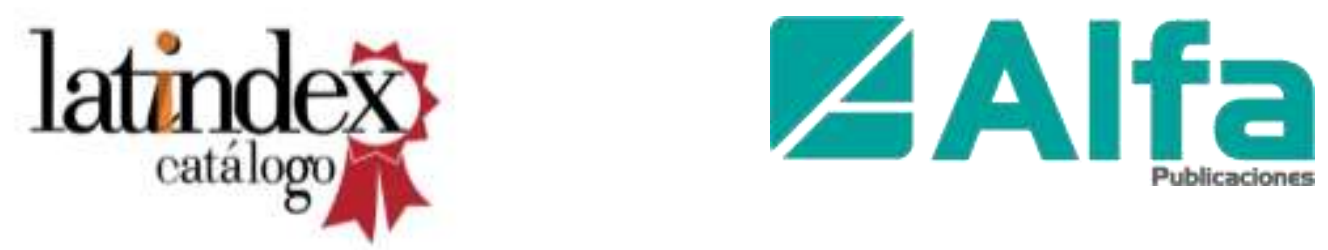

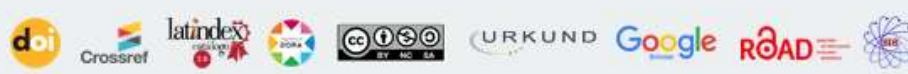
DLatinREV

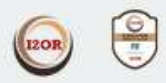

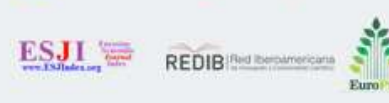

$\underbrace{}_{\text {wizdom.ai }} \mathrm{O}_{\text {OpenAIRE }}^{+}$

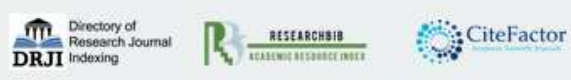

